\title{
The three-wave coupling coefficients for a cold magnetized plasma
}

\author{
L. Stenflo and G. Brodin \\ Department of Physics, Umeå University, SE-901 87 Umeå, Sweden
}

\begin{abstract}
The resonant interaction between three waves in a uniform magnetized plasma is considered. Using the somewhat inaccessible result of the general theory we deduce the explicit expressions for the coupling coefficients of a cold magnetized two-component plasma.
\end{abstract}




\section{INTRODUCTION}

The physics of nonlinear waves is a rapidly developing research field which has recently received increased attention (e.g. Shukla 2004; Stenflo 2004; Onishchenko et al 2004; Vladimirov and Yu 2004; Wu and Chao 2004; Shukla and Stenflo 2005a,b; Azeem and Mirza 2005; Mendonca et al. 2005; Marklund and Shukla 2005). Although there are general formalisms to treat such phenomena, there is also a need to have access to reliable explicit expressions for specific cases. As a particular example, Brodin and Stenflo $(1988 ; 1990)$ considered the resonant interaction between three MHD waves in

a plasma. Starting from the standard MHD theory they thus derived the coupling coefficients. However, the textbook MHD equations are not able to correctly treat the nonlinear interaction between three Alfvén waves (c.f. Shukla and Stenflo 2005b). In the present paper we are therefore going to reconsider the general nonlinear interaction between three waves in a cold, magnetized, two-component plasma, in order to derive the explicit expressions for the coupling coefficients. Such expressions have previously been presented for a one-component plasma (Stenflo 1973), but, due to algebraic difficulties, never before for a two-component plasma.

\section{RESULTS}

Considering the resonant interaction between three waves with frequencies $\omega_{j}(j=1,2,3)$ and wave vectors $\mathbf{k}_{j}$, we assume that the matching conditions

$$
\omega_{3}=\omega_{1}+\omega_{2}
$$

and

$$
\mathbf{k}_{3}=\mathbf{k}_{1}+\mathbf{k}_{2}
$$

are satisfied. The development of, for example, the z-components $\left(E_{j z}\right)$ of the wave electric field amplitudes is then governed by the three coupled bilinear equations (e.g. Stenflo 1994)

$$
\begin{aligned}
& \frac{d E_{1 z}^{*}}{d t}=\alpha_{1} E_{2 z} E_{3 z}^{*} \\
& \frac{d E_{2 z}^{*}}{d t}=\alpha_{2} E_{1 z} E_{3 z}^{*}
\end{aligned}
$$

and

$$
\frac{d E_{3 z}}{d t}=\alpha_{3} E_{2 z} E_{1 z}
$$

where the z-axis is along the external magnetic field $\left(B_{0} \widehat{\mathbf{z}}\right)$, the star denotes complex conjugate, $\alpha_{j}$ are the coupling coefficients, $d / d t=\partial / \partial t+\mathbf{v}_{g j} \cdot \nabla+\nu_{j}$ where $\mathbf{v}_{g j}$ is the group velocity of wave $j$, and $\nu_{j}$ accounts for the linear damping rate. The general formula for $\alpha_{j}$ for a hot magnetized plasma has been derived previously (e.g. Stenflo and Larsson 1977; Stenflo 1994) and it is therefore here only presented in Appendix A. Although, in principle, it covers all interaction mechanisms in uniform plasmas, it is not easy to apply it directly to, for example, Alfvén waves in a cold 
two-component plasma. However, after some straightforward, but rather lengthy evaluation of the formula in Appendix A, we can finally write $\alpha_{j}$ in the comparatively simple form

$$
\alpha_{1,2}=\frac{M_{1,2}}{\partial D\left(\omega_{1,2}, \mathbf{k}_{1,2}\right) / \partial \omega_{1,2}} C
$$

and

$$
\alpha_{3}=-\frac{M_{3}}{\partial D\left(\omega_{3}, \mathbf{k}_{3}\right) / \partial \omega_{3}} C
$$

where

$$
\begin{aligned}
& C=\sum_{\sigma} \frac{q \omega_{p}^{2}}{m \omega_{1} \omega_{2} \omega_{3} k_{1 z} k_{2 z} k_{3 z}}\left[\frac{\mathbf{k}_{1} \cdot \mathbf{K}_{1}}{\omega_{1}} \mathbf{K}_{2} \cdot \mathbf{K}_{3}^{*}+\frac{\mathbf{k}_{2} \cdot \mathbf{K}_{2}}{\omega_{2}} \mathbf{K}_{1} \cdot \mathbf{K}_{3}^{*}+\frac{\mathbf{k}_{3} \cdot \mathbf{K}_{3}^{*}}{\omega_{3}} \mathbf{K}_{1} \cdot \mathbf{K}_{2}-\right. \\
& \left.\frac{i \omega_{c}}{\omega_{3}}\left(\frac{k_{2 z}}{\omega_{2}}-\frac{k_{1 z}}{\omega_{1}}\right) \mathbf{K}_{3}^{*} \cdot\left(\mathbf{K}_{1} \times \mathbf{K}_{2}\right)\right] \\
& \mathbf{K}=-\left[\mathbf{k}_{\perp}+i \frac{\omega_{c}}{\omega} \mathbf{k} \times \widehat{\mathbf{z}}+\left(\frac{\sum i \frac{\omega_{c}}{\omega} \frac{\omega_{p}^{2}}{\omega^{2}-\omega_{c}^{2}}}{1-\frac{k^{2} c^{2}}{\omega^{2}}-\sum \frac{\omega_{p}^{2}}{\omega^{2}-\omega_{c}^{2}}}\right)\left(\mathbf{k} \times \widehat{\mathbf{z}}-i \frac{\omega_{c}}{\omega} \mathbf{k}_{\perp}\right)\right] \frac{\left(1-\frac{k_{\perp}^{2} c^{2}}{\omega^{2}}-\sum \frac{\omega_{p}^{2}}{\omega^{2}}\right) \omega^{4}}{\left(\omega^{2}-\omega_{c}^{2}\right) k_{\perp}^{2} c^{2}}+k_{z} \widehat{\mathbf{z}} \\
& D(\omega, \mathbf{k})=\left(1-\frac{k^{2} c^{2}}{\omega^{2}}-\sum \frac{\omega_{p}^{2}}{\omega^{2}-\omega_{c}^{2}}\right)\left[\left(\left(1-\frac{k_{z}^{2} c^{2}}{\omega^{2}}-\sum \frac{\omega_{p}^{2}}{\omega^{2}-\omega_{c}^{2}}\right)\left(1-\frac{k_{\perp}^{2} c^{2}}{\omega^{2}}-\sum \frac{\omega_{p}^{2}}{\omega^{2}}\right)-\right.\right. \\
& \left.\frac{k_{\perp}^{2} k_{z}^{2} c^{4}}{\omega^{4}}\right]-\left(\sum \frac{\omega_{p}^{2} \omega_{c}}{\omega\left(\omega^{2}-\omega_{c}^{2}\right)}\right)^{2}\left(1-\frac{k_{\perp}^{2} c^{2}}{\omega^{2}}-\sum \frac{\omega_{p}^{2}}{\omega^{2}}\right)
\end{aligned}
$$

and

$$
M_{j}=\left(1-\frac{k_{j}^{2} c^{2}}{\omega_{j}^{2}}-\sum \frac{\omega_{p}^{2}}{\omega_{j}^{2}-\omega_{c}^{2}}\right)\left(1-\frac{k_{j z}^{2} c^{2}}{\omega_{j}^{2}}-\sum \frac{\omega_{p}^{2}}{\omega_{j}^{2}-\omega_{c}^{2}}\right)-\left(\sum \frac{\omega_{p}^{2} \omega_{c}}{\omega_{j}\left(\omega_{j}^{2}-\omega_{c}^{2}\right)}\right)^{2}
$$

where $k=\left(k_{z}^{2}+k_{\perp}^{2}\right)^{1 / 2}, \mathbf{k}_{\perp}$ is the perpendicular (to $\widehat{\mathbf{z}}$ ) part of the wave-vector, $\omega_{p}$ is the plasma frequency ( $\omega_{p e}$ for the electrons and $\omega_{p i}$ for the ions), $\omega_{c}=q B_{0} / m$ is the cyclotron frequency, $q$ and $m$ are the particle charge and mass, and $c$ is the speed of light in vacuum. For notational convenience, the subscript $\sigma$ denoting the various particle species has been left out in the above formulas. We stress that no approximations have to be used to derive the expressions (4)-(8) which thus are quite general for the case of three wave interactions in a cold magnetized two-component plasma. It can also be verified that (4) agrees with the coupling coefficients for a magnetized one-component (Stenflo 1973; 1994) plasma.

Equations (3a,b,c), with (4), significantly improve the (approximate) equations in the previous work by Brodin and Stenflo (1988) for the case when the plasma is cold. Thus, although the main emphasis in that work was on the coupling between Alfvén waves and magnetosonic waves where useful results were derived, it was also mentioned that there is no coupling between Alfvén waves in the MHD limit. The present paper shows however that this is not true. Thus there is a non-zero 
interaction between, for example, one dispersive Alfvén pump wave (Shukla and Stenflo 2005b) and two inertial Alfvén waves characterized by

$$
\omega_{1,2} \simeq \frac{k_{1,2 z} V_{A}}{1+k_{1,2 \perp}^{2} \lambda_{e}^{2}}
$$

where $V_{A}$ is the Alfvén velocity and $\lambda_{e}=c / \omega_{p e}$. In the particular case when $E_{z}$ is zero for one of the waves, it is of course straightforward to use other variables, e.g. $E_{x}$ instead of $E_{z}$, to derive expressions similar to those above.

\section{CONCLUSIONS}

In the present paper we have improved the approximate results for three wave interactions in an MHD plasma (Brodin and Stenflo 1988) and found the explicit expressions for the coupling coefficients for wave interactions in a cold magnetized two-component plasma. Our coupling coefficient $C$ can thus be used as a starting point (see for example Appendix B) of any estimate of the coupling strength where the interaction between any kind of waves (Alfvén waves, whistler waves, etc.) in a cold plasma has to be considered. It can also be useful in interpretations of stimulated scattering of electromagnetic waves in space plasmas (e.g. Stenflo 1999; Kuo 2001; 2003). In the latter case we refer the reader to a short historical account of stimulated electromagnetic emissions in the ionosphere (Stenflo 2004).

\section{APPENDIX A}

When calculating the coupling coefficients, it turns out that they contain a common factor V. It is then possible to write the three coupled equations as

$$
\frac{d W_{1,2}}{d t}=-2 \omega_{1,2} \operatorname{ImV}
$$

and

$$
\frac{d W_{3}}{d t}=2 \omega_{3} \operatorname{ImV}
$$

where $W=\varepsilon_{0} \mathbf{E}^{*} \cdot(1 / \omega) \partial\left(\omega^{2} \boldsymbol{\varepsilon}\right) \mathbf{E}$ is the wave energy $\boldsymbol{\varepsilon}$ is the usual textbook dielectric tensor, and $\mathrm{ImV}$ stands for the imaginary part of $\mathrm{V}$ where (Stenflo and Larsson 1977; Stenflo 1994)

$$
\begin{gathered}
\mathrm{V}=\sum_{s} m \int d \mathbf{v} \mathbf{F}_{0}(\mathbf{v}) \sum_{\substack{p_{1}+p_{2}=p_{3} \\
p_{j}=0, \pm 1, \pm 2, \ldots}} I_{1}^{p_{1}} I_{2}^{p_{2}} I_{3}^{-p_{3}}\left[\frac{\mathbf{k}_{1} \cdot \mathbf{u}_{1 p_{1}}}{\omega_{1 d}} \mathbf{u}_{2 p_{2}} \cdot \mathbf{u}_{3 p_{3}}^{*}+\frac{\mathbf{k}_{2} \cdot \mathbf{u}_{2 p_{2}}}{\omega_{2 d}} \mathbf{u}_{1 p_{1}} \cdot \mathbf{u}_{3 p_{3}}^{*}+\frac{\mathbf{k}_{3} \cdot \mathbf{u}_{3 p_{3}}^{*}}{\omega_{3 d}} \mathbf{u}_{1 p_{1}} \cdot \mathbf{u}_{2 p_{2}}\right. \\
\left.\quad-\frac{i \omega_{c}}{\omega_{3 d}}\left(\frac{k_{2 z}}{\omega_{2 d}}-\frac{k_{1 z}}{\omega_{1 d}}\right) \mathbf{u}_{3 p_{3}}^{*} \cdot\left(\mathbf{u}_{1 p_{1}} \times \mathbf{u}_{2 p_{2}}\right)\right]
\end{gathered}
$$

where $\omega_{j d}=\omega_{j}-k_{j z} v_{z}-p_{j} \omega_{c}, I_{j}\left(=\exp \left(i \theta_{j}\right)\right)=\left(k_{j_{x}}+i k_{j y}\right) / k_{j \perp}$, and the velocity $\mathbf{u}_{j p_{j}}$ satisfies

$$
\omega_{j d} \mathbf{u}_{j p_{j}}+i \omega_{c} \widehat{\mathbf{z}} \times \mathbf{u}_{j p_{j}}=\frac{i q}{m \omega_{j}}\left\{\omega_{j d} J_{p_{j}} \mathbf{E}_{j}+\left[\left(v_{z} E_{j z}+\frac{p_{j} \omega_{c}}{k_{j \perp}^{2}} \mathbf{k}_{j \perp} \cdot \mathbf{E}_{j \perp}\right) J_{p_{j}}+\right.\right.
$$




$$
\left.\left.\frac{i v_{\perp} \omega_{c}}{k_{j \perp}^{2}}\left(\widehat{\mathbf{z}} \times \mathbf{k}_{j}\right) \cdot \mathbf{E}_{j} \frac{d}{d v_{\perp}} J_{p_{j}}\right] \mathbf{k}_{j}\right\}
$$

where $J_{p_{j}}=J_{p_{j}}\left(k_{j \perp} v_{\perp} / \omega_{c}\right)$ denotes a Bessel function of order $p_{j}$.

\section{APPENDIX B}

The limit when $\omega$ is much smaller than $\omega_{c i}$ is of special interest. In that case, we approximate (6) by

$$
\mathbf{K}_{e} \simeq-\frac{i \omega}{\omega_{c e}} \frac{\left(1+k_{\perp}^{2} \lambda_{e}^{2}\right)}{k_{\perp}^{2} \lambda_{e}^{2}} \mathbf{k} \times \widehat{\mathbf{z}}+k_{z} \widehat{\mathbf{z}}
$$

and

$$
\mathbf{K}_{i} \simeq-\frac{i \omega}{\omega_{c i}} \frac{\left(1+k_{\perp}^{2} \lambda_{e}^{2}\right)}{k_{\perp}^{2} \lambda_{e}^{2}}\left[\mathbf{k} \times \widehat{\mathbf{z}}-\frac{i \omega}{\omega_{c i}} \mathbf{k}_{\perp}\right]
$$

We note that the ion contributions dominate the first three terms in (5), whereas the electron contributions are most important for the fourth term in (5). As a result we have a non-zero coupling coefficient $C$ for the particular case of interaction between three Alfvén waves, in contrast to what one obtains from the too simplified textbook MHD equations (Brodin and Stenflo 1988; where $C_{A A A}$ was zero).

[1] Azeem M. and Mirza A. M. 2005 Phys. Plasmas 12, 052306.

[2] Brodin G. and Stenflo L. 1988 J. Plasma Phys. 39, 277.

[3] Brodin G. and Stenflo L. 1990 Contr. Plasma Phys. 30, 413.

[4] Kuo S. P. 2001 J. Plasma Phys. 66, 315.

[5] Kuo S. P. 2003 J. Plasma Phys. 69, 529.

[6] Marklund M. and Shukla P. K. 2005 Rev. Mod. Phys. To be published.

[7] Mendonca J. T., Serbeto A., Bingham R. and Shukla P. K. 2005 J. Plasma Phys. 71, 119.

[8] Onishchenko O.G., Pokhotelov O.A., Sagdeev R.Z., Shukla P.K. and Stenflo L. 2004 Nonl. Proc. Geophys. 11, 241.

[9] Shukla P.K. (Ed.) 2004 Nonlinear Physics in Action, Physica Scripta T113.

[10] Shukla P.K. and Stenflo L. (Eds) 2005a Modern Plasma Science, Physica Scripta T116.

[11] Shukla P.K. and Stenflo L. 2005b Phys. Plasmas 12, 084502.

[12] Stenflo L. 1973 Planet. Space Sci. 21, 391.

[13] Stenflo L. and Larsson J. 1977 in Plasma Physics: Nonlinear Theory and Experiments (Ed. H. Wilhelmsson) Plenum, New York, p. 152.

[14] Stenflo L. 1994 Phys. Scripta T50, 15.

[15] Stenflo L. 1999 J. Plasma Phys. 61, 129.

[16] Stenflo L. 2004 Phys. Scripta T107, 262. 
[17] Vladimirov S. V. and Yu M. Y. 2004 Physica Scripta T113, 32.

[18] Wu D. J. and Chao J. K. 2004 Nonl. Proc. Geophys. 11, 631. 